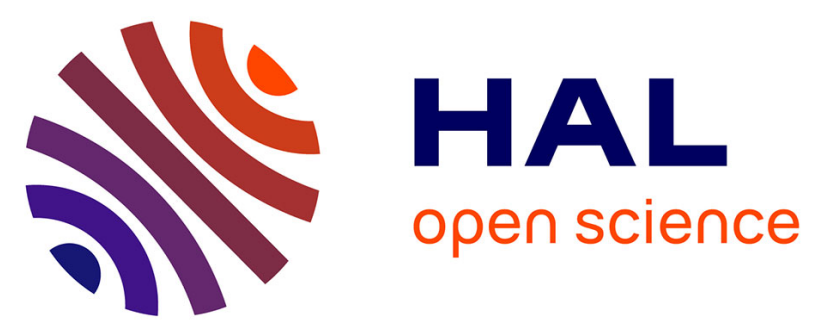

\title{
Allergie aux protéines du lait de vache : guide pratique de la réintroduction des protéines du lait de vache : quand, comment réintroduire
}

E. Bidat, A. Deschildre, A. Lemoine, G. Benoist, J. Valleteau de Moulliac, C. Tressol, K. Garcette, A. Juchet, E. Michaud, J. Languepin, et al.

\section{To cite this version:}

E. Bidat, A. Deschildre, A. Lemoine, G. Benoist, J. Valleteau de Moulliac, et al.. Allergie aux protéines du lait de vache : guide pratique de la réintroduction des protéines du lait de vache : quand, comment réintroduire. Revue francaise d'allergologie, 2019, 59 (1), pp.41-53. 10.1016/j.reval.2018.11.006 . hal02055748

\section{HAL Id: hal-02055748 \\ https: / hal.sorbonne-universite.fr/hal-02055748}

Submitted on 29 Aug 2019

HAL is a multi-disciplinary open access archive for the deposit and dissemination of scientific research documents, whether they are published or not. The documents may come from teaching and research institutions in France or abroad, or from public or private research centers.
L'archive ouverte pluridisciplinaire HAL, est destinée au dépôt et à la diffusion de documents scientifiques de niveau recherche, publiés ou non, émanant des établissements d'enseignement et de recherche français ou étrangers, des laboratoires publics ou privés. 


\title{
Allergie aux protéines du lait de vache : guide pratique de la réintroduction des protéines du lait de vache : quand, comment réintroduire
}

\section{Cow's milk protein allergy: A practical guide to the reintroduction of cow's milk proteins-when and how it should be reintroduced}

\author{
E. Bidat ${ }^{\mathrm{a}, *}$, A. Deschildre ${ }^{\mathrm{b}}$, A. Lemoine $^{\mathrm{c}}$, G. Benoist ${ }^{\mathrm{d}}$, J. Valleteau de Moulliac $^{\mathrm{a}}$, C. Tressol $^{\mathrm{e}}$, \\ K. Garcette ${ }^{\mathrm{f}}$, A. Juchet ${ }^{\mathrm{g}}$, E. Michaud ${ }^{\mathrm{h}}$, J. Languepin ${ }^{\mathrm{i}}$, T. Lamireau ${ }^{\mathrm{j}}$, A. Lachaux ${ }^{\mathrm{k}}$, P. Tounian ${ }^{\mathrm{c}}$ \\ ${ }^{a}$ Cabinet médical, 60, boulevard Emile-Augier, 75116 Paris, France \\ ${ }^{\mathrm{b}}$ Hôpital Jeanne-de-Flandre, CHRU de Lille, 59000 Lille, France \\ ${ }^{\mathrm{c}}$ Nutrition et gastroentérologie pédiatriques, hôpital Trousseau, 26, avenue du Dr-Arnold-Netter, 75012 Paris, France \\ ${ }^{\mathrm{d}}$ Service de pédiatrie générale et HDJ de pneumo-allergologie, CHU Ambroise-Paré, AP-HP, 9, avenue Charles-de-Gaulle, 92100 Boulogne-Billancourt, France \\ ${ }^{\mathrm{e}}$ Hôpital Ambroise-Paré, AP-HP, 9, avenue Charles-de-Gaulle, 92104 Boulogne cedex, France \\ ${ }^{\mathrm{f}}$ Centre médical spécialisé de l'enfant et de l'adolescent, 17, rue Froment, 75011 Paris, France \\ ${ }^{\mathrm{g}}$ Hopital des Enfants, 31059 Toulouse cedex 9, France \\ ${ }^{\mathrm{h}}$ Hôpital Estaing, CHU de Clermont-Ferrand, 1, place Lucie-et-Raymond-Aubrac, 63100 Clermont-Ferrand, France \\ ${ }^{i}$ Hépato-gastroentérologie et nutrition pédiatrique, département de pédiatrie médicale, hôpital Mère-Enfant, CHU de Limoges, 8, avevue D.-Larrey, 87000, France \\ ${ }^{\mathrm{j}}$ Unité de gastroentérologie, hépatologie et nutrition pédiatrique, hôpital des Enfants, place Amelie-Raba-Léon, 33077 Bordeaux, France \\ ${ }^{\mathrm{k}}$ Service de gastroentérologie, hépatologie et nutrition pédiatrique, HFME du CHU de Lyon, 59, boulevard Pinel, 69677 Bron, France
}

\section{Résumé}

Une fois le diagnostic d'allergie aux protéines du lait de vache (APLV) effectué se posent les questions du moment et des modalités de la réintroduction. Les connaissances actuelles permettent de proposer des prises en charge adaptées à la diversité des tableaux cliniques de l'APLV. Après avoir rappelé les acquis récents dans les différentes formes d'APLV, envisagé les connaissances actuelles sur l'immunothérapie au lait de vache, nous proposerons des attitudes pratiques sur les indications et les modalités de réintroduction dans les APLV de forme non IgE médiée et IgE médiée. Cet article reflète l'analyse récente de la littérature et les expériences des différents contributeurs.

Mots clés : Immunothérapie ; Allergie alimentaire ; Lait de vache ; Enfant ; Test de provocation par voie orale

\section{Abstract}

Once cow's milk protein allergy (CMA) has been diagnosed, the question is when and how it should be reintroduced. Current knowledge makes it possible to offer treatments suited to the diversity of clinical presentations. After a summary analysis of recent advances in different forms of CMA, based on current knowledge about cow's milk immunotherapy, we propose practical approaches for indications and reintroduction methods in both non-IgE-mediated and IgE-mediated CMA. This article reflects a recent literature analysis as well as the experiences of various contributors.

\footnotetext{
* Auteur correspondant.

Adresse e-mail : e.bidat@orange.fr (E. Bidat).
} 


\section{Introduction}

L'allergie aux protéines du lait de vache (APLV) est très fréquente dans les premières années de vie [1]. Elle débute le plus souvent dans les 6 premiers mois, rarement après 12 mois. Dans la première année de vie, elle toucherait 1,8 à 7,5\% des enfants, selon les séries et les pays. Cette disparité de prévalence est en partie liée aux critères diagnostiques retenus [2,3].

Une fois le diagnostic d'APLV effectué, le régime d'éviction institué, se posent les questions du moment et des modalités de la réintroduction des produits à base de protéines du lait de vache.

Les connaissances actuelles permettent de proposer des prises en charge adaptées à la diversité des tableaux cliniques de l'APLV et à leur évolution sous régime d'éviction, dans le cadre d'une prise en charge personnalisée.

Après avoir rappelé les acquis récents dans les différentes formes d'APLV, envisagé les connaissances actuelles sur l'immunothérapie au lait de vache, nous proposerons des attitudes pratiques sur les indications et les modalités de réintroduction dans les APLV de forme non IgE médiée et $\operatorname{IgE}$ médiée.

Cet article reflète l' analyse récente de la littérature et les expériences des différents contributeurs, allergologues pédiatres, gastroentérologues pédiatres, pédiatres généralistes, diététicienne.

La nécessité de systématisation entraînera inévitablement des simplifications. Nous essaierons de rester proche de la pratique clinique et d'envisager la majorité des situations que rencontre le médecin prenant en charge des enfants avec APLV. Nous n'aborderons pas la prise en charge des œsophagites à éosinophiles qui sort du domaine de la pratique courante.

\section{Acquis récents sur les APLV et leurs traitements}

\subsection{Les différentes formes d'allergies aux protéines du lait de vache}

On distingue différents types d'APLV en fonction du délai d'apparition des manifestations allergiques : immédiates et semi-retardées ou retardées. Dans les APLV immédiates ou IgE médiées, les symptômes apparaissent habituellement dans les minutes suivant l'ingestion, au maximum dans les 2-3 heures. Dans les APLV semi-retardées ou retardées ou non IgE médiées, les symptômes sont plus tardifs, au moins 2 heures après l'ingestion, souvent plusieurs jours.

Dans les formes IgE médiées, le spectre des signes est très large. Il s'étend de la simple urticaire péribuccale à la réaction anaphylactique pouvant mettre en jeu le pronostic vital.

Dans les formes non IgE médiées, les symptômes sont parfois difficiles à relier à la prise de l'allergène et sont souvent digestifs ou cutanés et non spécifiques. Ils vont du saignement rectal bien toléré observé dans la proctocolite allergique, aux vomissements itératifs sévères conduisant au choc hypovolémique dans le syndrome d'entérocolite induite par les protéines alimentaires (SEIPA). Il est important de noter que les études européennes montrent que les APLV non IgE médiées se présentent le plus
Tableau 1

Présentation des APLV dans les premières années de vie (d'après [1]).

Allergie non IgE médiée modérée

Habituellement 2-72 heures après l'ingestion des PLV (biberon ou via le lait de mère)

Généralement plusieurs signes sont présents

La résistance des signes au traitement symptomatique, notamment de l'eczéma, des coliques ou du reflux gastro-œsophagien, renforce la probabilité de l'allergie

Signes gastrointestinaux

«Coliques»

Reflux

Refus de l'aliment ou aversion

Selles molles et fréquentes

Constipation

Inconfort abdominal, flatulences

Sang ou mucus dans les selles chez un enfant bien portant

Peau

Prurit

Rash non spécifiques

Eczéma

Allergie non IgE médiée sévère

Habituellement 2-72 heures après l'ingestion des PLV (biberon ou via le lait de mère)

Un ou plusieurs de ces signes sévères persistent

Signes gastrointestinaux

Diarrhée

Vomissements

Douleurs abdominales (chez un enfant en âge de s'exprimer)

Refus de l'aliment ou aversion

Sang ou mucus dans les selles

Selles irrégulières et inconfortables

Mauvaise prise de poids

Peau

Eczéma sévère (SCORAD > 40)

Allergie IgE médiée non sévère

Habituellement dans les minutes après l'ingestion des PLV (parfois jusque deux heures après l'ingestion d'un biberon ou via le lait de mère) Peau

Prurit, rash erythèmateux, urticaire localisée

Angio-œdème sans signe respiratoire

Poussées d'eczéma ou eczéma persistant localisé

Digestif

Douleurs abdominales (chez un enfant en âge de s'exprimer)

Reflux gastro-oesophagien et vomissements

Diarrhée

Respiratoire

Rhinite aiguë

Conjonctivite

Allergie IgE médiée sévère : anaphylaxie

Réaction immédiate avec des signes respiratoires et/ou cardiovasculaires et/ou cutanés généralisés

souvent avec des signes légers à modérés et chroniques [1,4] (Tableaux 1 et 2).

La classification entre formes IgE et non IgE médiées correspond à la majorité des situations. Néanmoins il existe des formes mixtes. Par exemple dans certains SEIPA ou eczémas, les manifestations peuvent être IgE médiées et non IgE médiées. En cas de forme mixte, la prise en charge prend en considération la forme potentiellement la plus sévère, c'est à dire IgE médiée. Il existe parfois une évolution d'une forme à une autre. Une allergie peut être initialement non IgE médiée avec des manifestations digestives légères, puis avec le temps et sous régime d'éviction, 
Tableau 2

Anaphylaxie (d'après [4]).

L'anaphylaxie est considérée comme hautement probable dans trois situations

La survenue rapide d'une urticaire, d'un œdème, d'un prurit, d'un flush et au moins un des deux signes suivants

Dyspnée, bronchospasme, hypoxémie

Hypotension, choc

L'exposition à un allergène connu du patient et survenue dans les minutes ou heures de deux des signes suivants

Urticaire généralisée, œdème, prurit, flush

Dyspnée, bronchospasme, hypoxémie

Hypotension, choc

Crampes abdominales, vomissements répétés

La survenue d'une hypotension artérielle dans les minutes ou heures après l'exposition à un allergène connu

1 mois -1 an : PAS $<70 \mathrm{~mm} \mathrm{Hg}$

$1-10$ ans : PAS $<70 \mathrm{~mm} \mathrm{Hg}+$ (2 fois l'âge)

11-17 ans : PAS $<90 \mathrm{~mm} \mathrm{Hg}$

PAS : pression artérielle systolique.

évoluer vers une forme IgE médiée avec risque de manifestations allergiques sévères. On estime que ce passage d'une forme non IgE médiée vers une forme IgE médiée concernerait 10-15\% des nourrissons, bien que l'on manque de données issues d'études de suivi de cohorte. Cette évolution possible de l'APLV est à connaître. Cela impose, avant d'envisager une réintroduction, de s'assurer de l'absence d'apparition d'IgE spécifiques, à l'aide de prick-test au lait et/ou par un dosage des IgE spécifiques du lait de vache. À l'opposé, mais exceptionnellement, il est décrit des passages de forme $\operatorname{IgE}$ médiées vers des formes non $\operatorname{IgE}$ médiées.

\subsection{Diagnostic}

L'objet du présent document n'est pas de reprendre la démarche diagnostique de l'APLV, qui est maintenant consensuelle $[5,6]$.

La base de l'enquête allergologique repose sur l'interrogatoire.

En cas de manifestations cliniques d'allure IgE médiée (Tableaux 1 et 2), l'enquête est complétée par des prick-tests au lait de vache et un dosage d'IgE spécifiques. Si l'histoire clinique et les tests sont cohérents, la probabilité diagnostique est suffisante pour engager un régime d'éviction d'au moins 6 mois. S'il existe une discordance entre histoire clinique et tests, il faut faire un test de provocation par voie orale (TPO) en milieu hospitalier afin de ne pas instituer un régime d'éviction inutile et potentiellement délétère [5].

En cas de manifestations cliniques d'allure non IgE médiées légères à modérées (Tableau 1), qui sont les formes les plus fréquentes, le diagnostic se fonde avant tout sur une épreuve d'éviction de tous les laits et produits laitiers fabriqués à partir de protéines entières du lait de vache, suivi d'une réintroduction à domicile 2 à 4 semaines plus tard. Il n'est pas utile lors de ce test diagnostique de rechercher un passage d'une forme non IgE médiée vers une forme IgE médiée en raison de la courte durée de l'éviction des PLV. Lors du régime d'éviction, le remplacement du lait infantile avec protéines entières du lait de vache par un hydrolysat poussé de protéines du lait de vache doit conduire à une amélioration franche (par exemple d'un eczéma) voire une disparition des signes cliniques (par exemple digestifs), qui doivent récidiver, le plus souvent sous la même forme clinique, lors de la réintroduction du lait. Si ceci n'est pas observé, le diagnostic d'APLV non IgE médiée est exclu. Si les signes s'améliorent sous régime et se reproduisent lors de la réintroduction, le diagnostic est alors établi avec certitude ; le régime d'éviction sera alors poursuivi pendant au moins 2-6 mois, en fonction du tableau clinique, avant de discuter une réintroduction à la recherche d'une guérison de l'APLV.

Dans les formes sévères d'allergie non IgE médiée, comme une entéropathie sévère avec cassure de la courbe de poids, c'est l'évolution, souvent spectaculaire, sous régime d'éviction qui fait le diagnostic [1]. La réintroduction n'est donc pas faite à 1 mois et se fait habituellement à l'âge habituel de guérison.

Pour le SEIPA, les critères diagnostiques sont parfaitement définis. Si le tableau clinique répond à ces critères stricts, la probabilité diagnostique est suffisante pour effectuer un régime d'éviction sans réintroduction à visée diagnostique (Tableaux 3 et 4). Si tous les critères diagnostiques ne sont pas réunis, il faut recourir au TPO selon des modalités particulières au SEIPA $[7,8]$.

Dans la prise en charge initiale des APLV non IgE médiées, quand il existe un eczéma ou l'association de signes d'APLV non IgE médiées et IgE médiées (Tableaux 1 et 2), il est souhaitable de compléter l'enquête par des prick-tests au lait de vache et/ou un dosage d'IgE spécifiques afin de ne pas passer à côté d'une forme mixte, IgE et non IgE médiée. Cette forme nécessite une prise en charge identique à celle des allergies IgE médiées.

Tableau 3

Critères diagnostiques du SEIPA aigu ( 1 critère majeur et $\geq 3$ critères mineurs) (d'après [7]).

Critère majeur

Vomissements répétés survenant 1 à 4 heures après l'ingestion de l'aliment suspect en l'absence de signe cutané ou respiratoire d'allergie IgE médiée

Critères mineurs

1. $\geq 2$ épisodes de vomissements répétés après la reprise de l'aliment suspect

2. Vomissements répétés 1-4 heures après l'ingestion d'un autre aliment

3. Léthargie lors d'une réaction

4. Pâleur marquée lors d'une réaction

5. Consultation d'urgence lors d'une réaction

6. Perfusion IV lors d'une réaction

7. Diarrhée dans les 24 heures (habituellement 5-10 h)

8. Hypotension

9. Hypothermie

Si un seul épisode de vomissement est survenu, un TPO est souvent nécessaire tant les gastroentérites sont fréquentes dans cette tranche d'âge. Un épisode aigu de SEIPA est résolutif en quelques heures, beaucoup plus rapidement qu'une gastroentérite

Une fois l'aliment suspect éliminé, le patient doit être asymptomatique et grandir normalement 
Tableau 4

Critères diagnostiques du SEIPA chronique (d'après [7]).

Vomissements et diarrhées répétés et progressifs, observés avec un aliment de consommation courante comme le lait

Déshydratation et acidose métabolique possibles dans les formes

sévères

Résolution des signes en quelques jours de régime d'exclusion

Réapparition des signes lors de la réintroduction

Vomissements en 1-4 heures

Diarrhée dans les 24 heures (habituellement 5-10 h)

En l'absence d'épreuve d'exclusion/réintroduction le diagnostic reste présumé

\section{Traitements, ce qui a changé}

Les différentes prises en charge des APLV sont détaillées dans le chapitre suivant. Nous aborderons ici ce qui est nouveau dans la prise en charge des APLV : l'immunothérapie orale (ITO).

\subsection{Un changement dans le paradigme du traitement des APLV IgE médiées}

La prise en charge de l'APLV a énormément évolué au cours des dernières années. Par le passé, le traitement reposait sur une éviction stricte du lait de vache sous toutes ses formes. Cette éviction était censée accélérer la guérison de l'allergie. Si elle reste la règle au moment du diagnostic, la persistance de l'APLV au-delà de 4-5 ans conduit à envisager une réintroduction des protéines du lait dans le régime de l'enfant sous formes cuite, crue, ou fermentée et selon une quantité établie lors d'une consultation spécialisée. On distingue différentes présentations de lait. Le lait dit cru, peut être naturel, pasteurisé ou UHT. Le lait cuit, peut être peu cuit $\left(40^{\circ}\right)$ et fermenté dans les yoghourts et certains desserts lactés, il peut être cuit $\left(70-80^{\circ}\right)$ dans les fromages. Le lait peut enfin être très cuit $\left(180^{\circ}\right)$, couplé à une matrice blé dans des gâteaux, comme par exemple les véritables Petit Beurre de $\mathrm{Lu}^{\circledR}$.

Des travaux, en particulier ceux de Nowak-Wegrzyn et al. [9], ont montré que dans les APLV IgE médiées persistantes au-delà de l'âge habituel de guérison, certains enfants toléraient le lait cuit avant de tolérer le lait cru. Dans ce cas, l'introduction du lait cuit accélérerait la guérison naturelle de l'APLV avec une tolérance de toutes les formes de lait, crues et cuites. Cette introduction, quand elle est faite progressivement, est appelée ITO. Les enfants tolérant le lait cuit ou le lait fermenté (laitages) ont des caractéristiques particulières, notamment une sensibilisation moindre au lait (taille du prick test, taux des IgE spécifiques à la caséine). Le répertoire épitopique reconnu par les IgE est moindre du fait du changement conformationnel des protéines induit par la cuisson du lait [10]. Le lait cuit est alors moins allergisant que le lait non cuit.

C'est aussi le cas pour l'association à une « matrice » blé qui diminue l'allergénicité [11]. Cette association idéale, blé et lait cuit, est retrouvée dans les gâteaux.

À la suite des travaux initiaux, de nombreux autres ont permis d'affiner la pratique de l'ITO pour le lait dans les allergies IgE médiées. Aucune étude ne s'est penchée sur l'ITO dans l'allergie non IgE médiée aux PLV, mais, par un raisonnement analogique, il est proposé des ITO suivant le même schéma [1].

L'ITO est une nouvelle possibilité de traitement chez les enfants dont l'APLV persiste [2].

\subsection{Introduction du lait par immunothérapie orale : quels objectifs?}

Chez l'enfant présentant une allergie alimentaire persistante, les expositions alimentaires accidentelles ne sont pas exceptionnelles. Elles sont à l'origine de réactions, parfois anaphylactiques. Le régime d'éviction est souvent difficile à suivre, parfois responsable de carences, il marginalise l'enfant dans sa vie sociale (école, cantine, goûter d'anniversaire, etc.). L'ITO a radicalement modifié la prise en charge de l'allergie alimentaire des enfants présentant une allergie persistante [11].

Une analyse des études publiées montre que la probabilité de développer une tolérance au lait est 10 fois plus grande chez les enfants qui reçoivent une ITO comparée à ceux qui sont en régime d'éviction prolongé. De même, la probabilité de développer une tolérance partielle est 5 fois plus élevée [12].

L'ITO accélère donc la guérison de l'APLV, limite le risque d'accident lors d'une prise accidentelle de petite quantité de lait de vache, facilite le régime alimentaire, et améliore globalement la qualité de vie.

\subsection{ITO : quelles conditions préalables?}

Les conditions requises sont une allergie bien identifiée, une dose réactogène connue, une éducation thérapeutique des parents (et de l'enfant selon l'âge), notamment pour la gestion de l'urgence (trousse d'urgence, plan d'action), une information sur les co-facteurs de risque de réaction et une adhésion de la famille.

Débuter une ITO passe donc le plus souvent par plusieurs consultations abordant et précisant ces points. Il importe d'évaluer le risque face au bénéfice attendu. Certaines situations sont peu compatibles avec l'initiation d'une ITO, a fortiori à domicile (Tableau 5).

Tableau 5

Chez ces enfants à haut risque une consultation spécialisée est conseillée afin de discuter l'attitude optimale (d'après [2]).

Antécédents de signes d'APLV avec manifestations anaphylactiques respiratoires, et/ou circulatoires et/ou digestives sévères

Réaction sévère lors de l'exposition à des quantités faibles de LV Asthme non contrôlé

Allergies alimentaires multiples

Antécédent d'anaphylaxie à un autre aliment

Absence de diminution de la sensibilisation IgE (prick-test, IgE spécifiques)

Forte sensibilisation IgE sans prise antérieure de LV (par exemple enfant allaité ou avec hydrolysat de Protéines de LV qui présente un eczéma sévère)

Parents qui ne sont pas capables de comprendre ou d'adhérer au protocole

APLV : allergie aux protéines du lait de vache ; LV : lait de vache 
Tableau 6

Équivalences en produits laitiers.

\begin{tabular}{|c|c|c|c|c|c|}
\hline \multirow{2}{*}{$\begin{array}{l}\text { Aliment } \\
\text { Lait } 1 / 2 \text { écrémé } 1 \text { biberon de } 250 \mathrm{~mL}\end{array}$} & \multirow{2}{*}{$\begin{array}{l}\text { Portion } \\
100 \mathrm{~mL} 250 \mathrm{~mL}\end{array}$} & \multicolumn{2}{|c|}{ Protéinespar portion } & \multicolumn{2}{|c|}{ Équivalence en lait (approximatif) } \\
\hline & & $3,2 \mathrm{~g}$ & $8 \mathrm{~g}$ & & \\
\hline Beurre & $100 \mathrm{~g} \quad 30 \mathrm{~g}$ & $0,7 \mathrm{~g}$ & $0,23 \mathrm{~g}$ & $=21,8 \mathrm{~mL}$ & $=6,5 \mathrm{~mL}$ \\
\hline 1 petite plaquette de beurre individuelle & $10 \mathrm{~g}$ & & & $=2,18 \mathrm{~mL}$ & \\
\hline Apéricube La Vache Qui Rit & $5,2 \mathrm{~g}$ & $0,6 \mathrm{~g}$ & & $=19 \mathrm{~mL}$ & \\
\hline 1 Petit Filou tub's & $40 \mathrm{~g}$ & $1,44 \mathrm{~g}$ & & $=45 \mathrm{~mL}$ & \\
\hline Danao fruité & $250 \mathrm{~mL}$ & $1,75 \mathrm{~g}$ & & $=55 \mathrm{~mL}$ & \\
\hline Kiri crème & $20 \mathrm{~g}$ & $1,8 \mathrm{~g}$ & & $=56 \mathrm{~mL}$ & \\
\hline Vache qui rit & $16,6 \mathrm{~g}$ & $1,9 \mathrm{~g}$ & & $=60 \mathrm{~mL}$ & \\
\hline 1 Petit Filou fruits Yoplait & $50 \mathrm{~g}$ & $2,65 \mathrm{~g}$ & & $=82 \mathrm{~mL}$ & \\
\hline Mini Yop & $100 \mathrm{~g}$ & $2,8 \mathrm{~g}$ & & $=87 \mathrm{~mL}$ & \\
\hline Actimel nature sucré & $100 \mathrm{~mL}$ & $3 \mathrm{~g}$ & & $=94 \mathrm{~mL}$ & \\
\hline Carré frais Gervais & $25 \mathrm{~g}$ & $3 \mathrm{~g}$ & & $=94 \mathrm{~mL}$ & \\
\hline Flamby Nestlé & $100 \mathrm{~g}$ & $3,1 \mathrm{~g}$ & & $=97 \mathrm{~mL}$ & \\
\hline 1Gervais aux fruits (Danonino) & $50 \mathrm{~g}$ & $3,2 \mathrm{~g}$ & & $=100 \mathrm{~mL}$ & \\
\hline 1 Danette crème dessert vanille & $125 \mathrm{~g}$ & $3 g$ & & $=117 \mathrm{~mL}$ & \\
\hline Velouté fruix Danone & $125 \mathrm{~g}$ & $4 \mathrm{~g}$ & & $=125 \mathrm{~mL}$ & \\
\hline 1 Activia saveur vanille & $125 \mathrm{~g}$ & $4,5 \mathrm{~g}$ & & $=140 \mathrm{~mL}$ & \\
\hline 1 petit suisse $10 \% \mathrm{MG}$ & $60 \mathrm{~g}$ & $5,4 \mathrm{~g}$ & & $=168 \mathrm{~mL}$ & \\
\hline 1/8 Camembert & $31 \mathrm{~g}$ & $6,4 \mathrm{~g}$ & & $=200 \mathrm{~mL}$ & \\
\hline Fromage blanc $3,2 \%$ & $100 \mathrm{~g}$ & $6,9 \mathrm{~g}$ & & $=215 \mathrm{~mL}$ & \\
\hline Emmental, comté, gruyère & $30 \mathrm{~g}$ & $8 g$ & & $=253 \mathrm{~mL}$ & \\
\hline
\end{tabular}

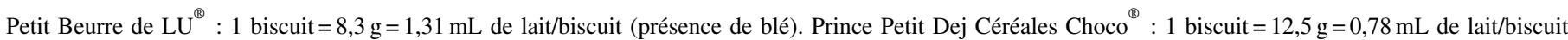

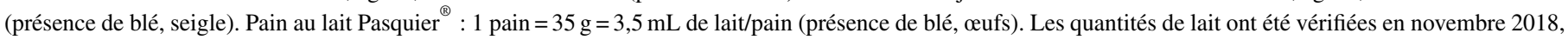

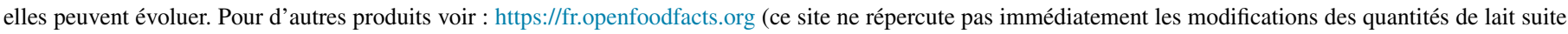
aux modifications des recettes ou des procédés de fabrication).

\subsection{Première étape : définir la dose réactogène}

\subsubsection{Test cutané et/ou IgE spécifiques ne remplacent pas un $T P O$}

S'il n'y a pas eu de prise accidentelle, il n'est pas possible à ce jour de fixer un seuil de test cutané ou d'IgE spécifiques qui permet d'éviter la pratique d'un TPO. De même il n'existe pas de corrélation entre la taille du test cutané ou le taux d'IgE spécifiques avec la réactivité clinique du patient au lait cuit ou cru [13]. Certains auteurs suggèrent qu'il peut être intéressant d'étudier la réactivité au lait cuit lors de TPO chez des patients dont les IgE lait de vache sont inférieures à 5 ou $10 \mathrm{kU} / \mathrm{L}$, ou dont les $\mathrm{IgE}$ caséines sont inférieures à $5 \mathrm{kU} / \mathrm{L}$ [14]. En effet, quand le patient a des IgE au-dessus de ces taux, les chances de succès du TPO au lait cuit seraient faibles. Ceci reste à discuter au cas par cas en fonction de nombreux facteurs. Il faut prendre en compte l'histoire clinique, la cinétique des tests cutanés et des IgE spécifiques, le taux des IgE totales, l'évolution des autres allergies alimentaires, les comorbidités (asthme en particulier) [15].

\subsubsection{Les prises accidentelles de lait ou laitage, une aide dans la prise en charge}

L'interrogatoire néglige souvent cette information, elle est pourtant capitale. Des parents qui ont donné un aliment contenant du lait à leur enfant allergique, quand celui-ci n'a pas présenté de réaction suite à cette ingestion, ne transmettent pas toujours spontanément l'information au médecin. Par contre si l'enfant a présenté une réaction, l'information est fournie ! En cas de prise accidentelle sans réaction, «l'erreur » ressentie par les parents devient une information et peut permettre d'éviter (ou de modifier) la réalisation d'un TPO. Il faut poser à chaque consultation la question de manière non culpabilisante pour obtenir l'information. Une question systématique dans le suivi d'un enfant ayant une APLV est indispensable : « votre enfant a-t-il pris, sans le faire exprès, un aliment contenant du lait». En cas de réponse positive, il faut essayer de chiffrer la quantité de lait consommé et le degré de cuisson du lait. Parfois, quand il s'agit d'un produit laitier, c'est facile. Pour un produit manufacturé, il est parfois plus difficile de préciser la quantité de lait contenue dans le produit consommé (Tableau 6). Le recours au site internet https://fr.openfoodfacts.org est d'une grande aide. Il suffit d'entrer le nom du produit consommé pour connaître la liste des ingrédients et parfois le pourcentage de lait. Ce site est d'une grande utilité, mais il faut comme toujours rester prudent, la mise à jour des informations, quand la composition d'un produit évolue, n'est pas immédiate.

\subsubsection{Dose réactogène définie par le TPO}

Pour débuter une ITO, à fortiori si les parents ne peuvent pas rapporter dans l'anamnèse une quantité de lait tolérée (cuit ou non), un TPO sous surveillance hospitalière est indispensable pour déterminer la dose seuil réactogène. Le choix du TPO, lait cuit ou cru, est fonction de l'histoire clinique, de l'âge de l'enfant, de l'évolution de la sensibilisation IgE, des éventuelles prises accidentelles.

Il existe deux exceptions :

- les APLV non IgE médiées légères à modérées, formes qui sont les plus fréquentes : on peut discuter la réintroduction 
et ses modalités, au domicile, après avoir vérifié l'absence d'apparition d'une sensibilisation allergique ;

- en cas de prise accidentelle de lait, la réaction (ou son absence) pour une quantité connue de PLV constitue un véritable TPO « sauvage».

Les protocoles de TPO, qui varient selon les équipes, suivent tous une même logique : augmentation progressive des doses toutes les 20-30 minutes et surveillance au décours de la dernière dose. La première dose de lait peut être plus importante si l'enfant a déjà consommé des petites quantités au domicile. Le protocole pour le SEIPA est particulier et n'est pas abordé ici [8].

Pour le TPO au lait cuit, on peut, par exemple pour les patients hautement allergiques, débuter avec les « Véritable Petit Beurre de $\operatorname{LU}^{\circledR}$ » $(1,31 \mathrm{~mL}$ de lait de vache par unité), qui seront fractionnés pour les premières doses. $\mathrm{Si} 4$ «Véritable Petit Beurre de LU $^{\circledR}$ " sont tolérés, le TPO est poursuivi avec des quantités croissantes de gâteaux industriels ou de gâteaux maison contenant des quantités connues de lait (Tableau 6). En cas de négativité du TPO avec ces produits cuits, l'élargissement du régime est alors poursuivi dans un deuxième temps par un TPO au lait cru.

\subsection{ITO en pratique}

\subsubsection{Pour qui?}

L'ITO avec le lait cuit est proposé uniquement aux patients qui ne réagissent pas à des doses faibles de lait cuit lors du premier TPO, en milieu hospitalier. Pour ceux qui tolèrent des doses significatives de lait, il est possible d' effectuer une ITO en ville par des praticiens expérimentés et disponibles, en relation avec le médecin qui prend en charge l'enfant.

Il est difficile de définir ce qu'est une dose « infime » ou « significative », chaque situation est à examiner au cas par cas en tenant compte de l'histoire, des examens complémentaires et du contexte familial. L'expérience de l'équipe prenant en charge l'ITO est aussi très importante.

La dose initiale, a fortiori au cabinet de ville ou à la maison, est toujours largement inférieure à celle à laquelle l'enfant a pu réagir lors du TPO hospitalier. Les conditions de réalisation des TPO sont très éloignées de celles de la vraie vie où de nombreux co-facteurs sont présents (fièvre, stress, effort, etc.), pouvant modifier la dose réactogène [16] (Tableau 7). Il est donc plus prudent de débuter l'ITO au lait cuit avec des doses de lait inférieures à la dernière ingérée lors du TPO, en pratique au moins l'avant-dernière dose tolérée, et habituellement de l'ordre du millilitre. Puis la progression sera d'autant plus rapide que la dose réactogène est élevée.

Chez les patients qui réagissent à une dose très faible de lait, il est préférable de sursoir à l'ITO, ou de pratiquer une augmentation de dose très lente en milieu hospitalier et spécialisé. Le risque de réaction est élevé. Une autre alternative est de maintenir durablement une prise quotidienne d'une très petite quantité de lait tolérée par le patient.

Dans certaines situations, dites à haut risque, une consultation spécialisée est conseillée afin de discuter l'attitude optimale
Tableau 7

Facteurs aggravant une réaction par allergie alimentaire et augmentant le risque d'anaphylaxie (d'après [16]).

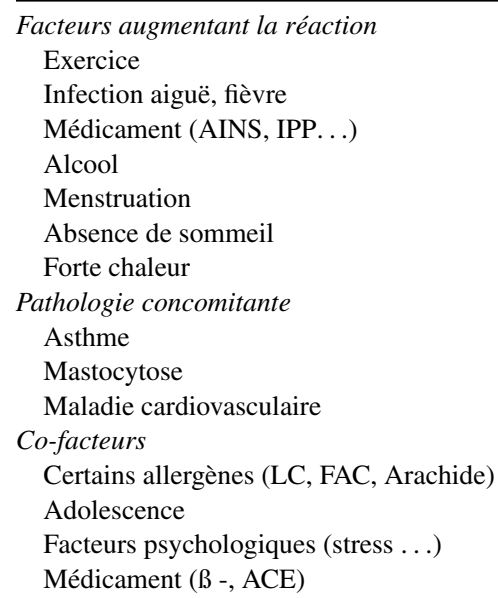

AINS : anti-inflammatoires non stéroïdiens ; IPP : inhibiteur de la pompe à protons ; LC :lait de chèvre. FAC : fruits à coque ; $\beta$ - : béta bloquant ; ACE : inhibiteur de l'enzyme de conversion.

(Tableau 5). Initier une ITO dans ce contexte passe par une évaluation du risque en regard du bénéfice, un échange avec la famille. Il faut rappeler que certains patients seront réfractaires, sans bénéfice de ce type de stratégie.

\subsection{2. À quel âge envisager une ITO ?}

Les recommandations Européennes de 2018 conseillent de n'envisager une ITO au lait qu'à partir de l'âge de 4-5 ans [18]. Cette recommandation se heurte au vécu des familles. Quand une famille constate que son enfant peut consommer une certaine quantité de lait, accidentellement ou lors d'un TPO, elle émet souvent le souhait de ne pas poursuivre une exclusion complète du lait et des produits laitiers. En pratique, en France, l'ITO au lait est donc souvent débutée plus tôt que dans les recommandations Européennes.

\subsubsection{Des objectifs adaptés à chaque situation : ne pas être trop ambitieux}

Au début de la pratique de l'ITO, l'objectif était d'obtenir la guérison intégrale de l'APLV. Cet objectif n'était pas celui du patient avec une allergie persistante, qui souhaitait surtout consommer de petites quantités de lait de vache dans des préparations, le plus souvent cuit, afin d'améliorer la qualité de vie et de limiter le risque de réaction en cas d'exposition accidentelle. En pratique clinique, il faut respecter les souhaits du patient et de sa famille. Il faut savoir faire un palier dans l'augmentation des doses, et pour certains patients prolonger ce palier, car ils ne désirent pas toujours consommer des aliments qui contiennent plus de produits laitiers [15].

Enfin, il existe des APLV « résistantes » à l'immunothérapie pour lesquelles il faudra revenir au régime d'éviction ou envisager dans un centre spécialisé d'autres stratégies en cours d'évaluation. 
Tableau 8

Échelle de lait chez les « grands ».

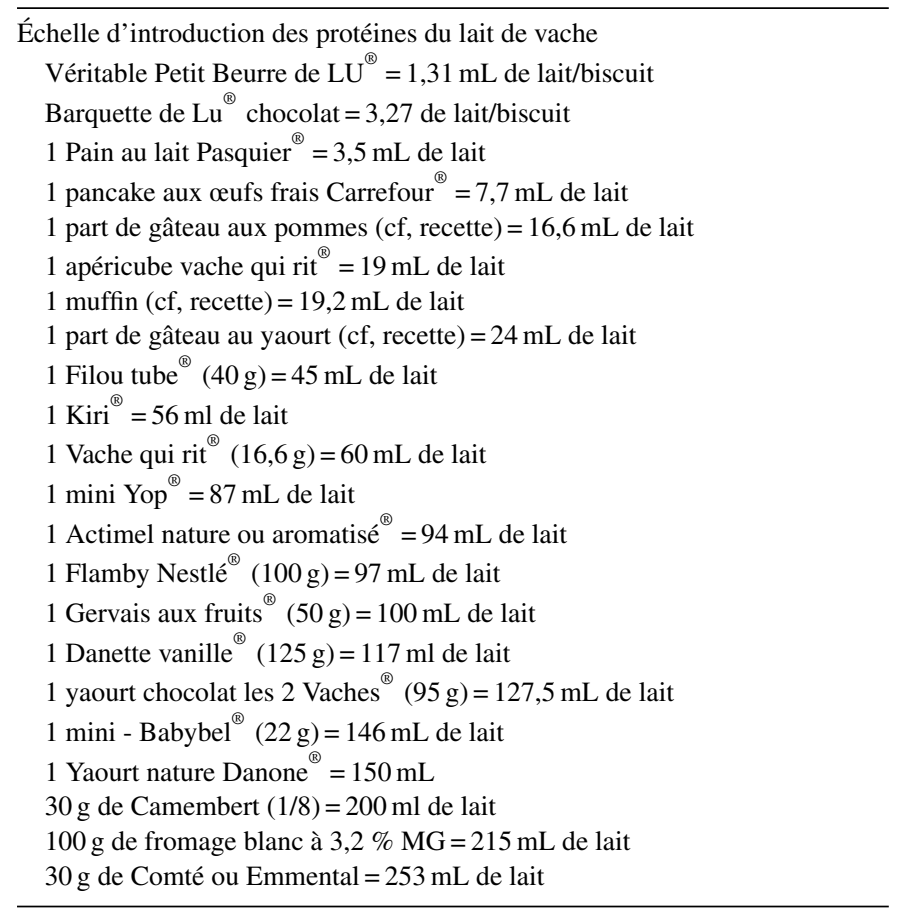

\subsubsection{L'échelle de lait commençant au lait cuit}

La progression des apports en protéines du lait de vache dans les ITO a été initiée par Carina Venter dès 2013, actualisée en $2017[1,17]$. Nous avons adapté pour la France cette « échelle de lait » [15]. Il existe d'autres échelles, reposant sur le même principe [19].

Une fois les petites quantités de lait cuit tolérées sous forme de biscuit (Tableau 8) l'augmentation progressive sous forme cuite est possible. L'échelle comprend plusieurs étapes avec des aliments à introduire dans un ordre précis, selon la teneur en lait et le niveau de cuisson. L'échelle de lait est surtout adaptée aux plus grands enfants ; chez les plus petits nous proposons une progression simplifiée (Tableau 9). Les recettes de gâteaux (Tableau 10) contiennent des quantités approchées de lait cuit par part, la répartition du lait pouvant ne pas être homogène. De plus, le degré de cuisson peut dépendre du four utilisé.

Pour les allergiques au lait et à l'œuf, une recette sans œuf est proposée. Les parents utilisent parfois les recettes prévues avec œufs en le remplaçant par un substitut. La prise de la préparation contenant du lait doit être quotidienne afin de ne pas perdre la tolérance acquise.

La durée de chaque étape est fonction de la situation initiale de l'enfant. Les éventuels incidents observés dans la prise des premiers produits avec le lait cuit ainsi que l'appétence de l'enfant pour ces produits guident la progression. Il est inutile et dangereux de contraindre un enfant qui refuse un produit, et la progression doit être stoppée. Il est préférable de prolonger un palier avec un aliment contenant moins de lait, mais bien accepté. Lorsque l'aliment d'une étape est bien toléré, et bien accepté, la progression est poursuivie. Elle doit être guidée par le médecin prescripteur.
Dans les formes les plus sévères d'APLV qui justifient un suivi spécialisé, la dose réactogène initiale de lait cuit, fixée par l'interrogatoire ou par le TPO, est maintenue constante au domicile. Les augmentations de dose se font en milieu hospitalier. Quand un nouveau palier est ainsi fixé, il est poursuivi au domicile. Dans cette situation, l'objectif peut être de maintenir une consommation quotidienne de lait en petite quantité pour protéger l'enfant du risque lié à l'ingestion de "lait caché », plutôt que de libérer la consommation du lait et des produits laitiers.

Si l'aliment d'une étape provoque une réaction allergique, les parents utilisent le protocole de traitement prévu et contacte le médecin guidant l'ITO avant de reprendre tout produit laitier ou contenant du lait (en particulier si la réaction est sévère). Si la réaction est jugée non sévère, l'enfant reprendra le palier précédent qui était toléré. Dans ce cas, le palier est généralement poursuivi pendant 15 à 30 jours, voire plus et selon l'avis du médecin.

Il faut signaler que les quantités des ingrédients, la composition nutritionnelle et/ou le grammage des produits indiqués dans l'échelle sont susceptibles d'évoluer au fil du temps et doivent donc être actualisés régulièrement. La quantité de lait peut varier pour un même produit de quelques millilitres, voire davantage (observation de variations de plus de $10 \%$ pour des fromages portions).

Des recommandations globales pour la conduite de l'ITO sont donc à remettre aux parents pour les accompagner au mieux (Tableau 11).

\subsection{Effets secondaires de l'ITO au lait de vache}

Les réactions anaphylactiques au lait de vache cuit sont toujours possibles. Elles sont plus fréquentes chez les enfants qui ont eu une réactivité pour des doses faibles lors du TPO initial. Si l'enfant présente lors de l'ITO des accidents anaphylactiques avec de petites doses de lait de vache cuit à la maison, le rapport bénéfice-risque impose de suspendre l'ITO ou d'envisager des nouvelles modalités thérapeutiques en cours d'évaluation.

Des œesophagites à éosinophiles sont observées chez 2,7 \% des patients bénéficiant d'une ITO principalement au lait de vache, à l'œuf, et à l'arachide [20]. Cette œsophagite à éosinophile est à rechercher par des questions systématiques : hypersalivation, reprise des symptômes du RGO, blocage alimentaire, boissons abondantes lors du repas.

Parmi des patients guéris de leur APLV suite à une ITO au lait de vache, jusqu'à $25,9 \%$ restaient allergiques au lait de chèvre ou de brebis [15]. Il est donc recommandé de vérifier, après guérison d'une APLV, l'acquisition de la tolérance au lait de chèvre ou de brebis par l'interrogatoire ou un TPO, en s'aidant éventuellement des tests cutanés.

Des anaphylaxies à l'effort, après prise de lait de vache, sont observées chez des enfants considérés « guéris » de leur allergie au lait mais au « repos » [15]. D'autres co-facteurs pourraient aboutir au même risque. Dans ce cas, on confirme une désensibilisation au lait de vache, rompue par un cofacteur, plutôt qu'une tolérance vraie et donc une guérison. 
Échelle de lait chez les «petits».

\begin{tabular}{|c|c|}
\hline Jours & Aliments à consommer (augmentation si pas de réaction) \\
\hline De j1 à j3 & $\begin{array}{l}1 / 2 \text { «Véritable Petit Beurre de } \mathrm{LU}^{\circledR} » \text { (il est possible de les écraser et les mélanger à de } \\
\text { la compote) }\end{array}$ \\
\hline De j4 à j6 & 1 «Véritable Petit Beurre de LU ${ }^{\circledR} »$ \\
\hline De j7 à j9 & 2 «Véritable Petit Beurre de LU ${ }^{\circledR} »$ \\
\hline De j10 à j12 & 3 «Véritable Petit Beurre de LU ${ }^{\circledR} »$ \\
\hline De j13 à j15 & 4 «Véritable Petit Beurre de LU ${ }^{\circledR} »$ \\
\hline De j16 à j25 & $\begin{array}{l}4 \text { «Véritable Petit Beurre de } \mathrm{LU}^{\circledR} » \text { par jour } \\
1 \text { noisette de beurre avec les légumes midi et soir }\end{array}$ \\
\hline De j26 à j28 & $\begin{array}{l}\text { Arrêt des «Véritable Petit Beurre de } \mathrm{LU}^{\circledR}{ }^{\circledR} » \\
1 / 2 \text { apéricube « la vache qui rit }{ }^{\circledR} \text { " par jour } \\
1 \text { noisette de beurre avec les légumes midi et soir }\end{array}$ \\
\hline De j29 à j31 & $\begin{array}{l}1 \text { apéricube « la vache qui rit }{ }^{\circledR} \text { » par jour } \\
1 \text { noisette de beurre avec les légumes midi et soir }\end{array}$ \\
\hline De j32 à j41 & $\begin{array}{l}4 \text { «Véritable Petit Beurre de } \mathrm{LU}^{\circledR} \text { " } \\
1 \text { apéricube « la vache qui rit }{ }^{\circledR} \text { par jour } \\
1 \text { noisette de beurre avec les légumes midi et soir }\end{array}$ \\
\hline De j42 à j44 & $\begin{array}{l}\text { Arrêt des «Véritable Petit Beurre de } \mathrm{LU}^{\circledR} \text { » et de l'apéricube « la vache qui rit }{ }^{\circledR} \text { " } \\
1 / 3 \text { de yaourt nature par jour } \\
1 \text { noisette de beurre avec les légumes midi et soir }\end{array}$ \\
\hline De j45 à j47 & $\begin{array}{l}1 / 2 \text { de yaourt nature par jour } \\
1 \text { noisette de beurre avec les légumes midi et soir }\end{array}$ \\
\hline De j48 à j50 & $\begin{array}{l}1 \text { de yaourt nature par jour } \\
1 \text { noisette de beurre avec les légumes midi et soir }\end{array}$ \\
\hline De j51 à j60 & $\begin{array}{l}1 \text { de yaourt nature par jour } \\
4 \text { «Véritable Petit Beurre de } \mathrm{LU}^{\circledR} \text { " } \\
1 \text { apéricube « la vache qui rit }{ }^{\circledR} \text { " par jour } \\
1 \text { noisette de beurre avec les légumes midi et soir }\end{array}$ \\
\hline De j61 à j63 & $\begin{array}{l}\text { Remplacer une mesure du lait habituel par une mesure de lait de croissance dans } \\
\text { chaque biberon } \\
1 \text { de yaourt nature par jour } \\
4 \text { «Véritable Petit Beurre de } \mathrm{LU}^{\circledR} \text { " } \\
1 \text { apéricube « la vache qui rit }{ }^{\circledR} \text { " par jour } \\
1 \text { noisette de beurre avec les légumes midi et soir }\end{array}$ \\
\hline À partir de j64 & Augmenter d'une mesure par biberon tous les 3 jours jusqu'au biberon complet \\
\hline
\end{tabular}

\subsection{Conseils pratiques pour l'ITO}

Le plus important avant d'envisager une ITO est d'évaluer la motivation de la famille, et la compréhension du protocole.

Il faut obtenir une adhésion de la famille et si possible de l'enfant en fonction de son âge. Lors du suivi d'enfants qui ont une allergie alimentaire (souvent une polyallergie), la question la plus importante est «qu'aimeriez-vous voir évoluer dans le régime ? ». Si l'enfant et la famille ne souhaitent pas de changements, il est inutile de proposer un bilan en vue d'une ITO.

L'ITO nécessite des ajustements dans l'augmentation des doses. Elle ne peut être proposée que chez des familles ayant compris son principe et maîtrisant parfaitement la gestion des réactions allergiques et de l'anaphylaxie dans les allergies $\operatorname{IgE}$ médiées. Il peut être justifié de suspendre une ITO chez des familles qui prennent des risques dans la progression des doses ou ne gèrent pas de manière optimale les incidents.

Les patients bénéficiant d'une ITO sont à suivre, à accompagner et à motiver au long cours. Ils doivent être informés du risque de réaction si la prise de $\mathrm{LV}$, même en petite quantité, est interrompue, même pour seulement une durée de l'ordre de quelques jours. C'est pourquoi, chez certains patients, le dégoût intense pour le lait rend impossible le projet d'ITO même pour des petites doses.

Certains co-facteurs peuvent modifier, lors de la prise de LV, le niveau de réactivité, des effets secondaires survenant alors plus facilement. Les principaux co-facteurs sont rappelés dans le Tableau 7 [16]. Ces co-facteurs doivent être connus des patients et de leur famille qui pratiquent une ITO, afin de moduler dans ces circonstances la prise des doses ingérées. En début de progression, il est prudent de surseoir pour 2 ou 3 jours à la prise de LV en cas d'infection et d'éviter une activité physique au décours de la prise (Tableau 7).

Enfin, le recours au lait cuit ne signifie pas nécessairement une meilleure tolérance, des effets secondaires y compris anaphylactiques pouvant être observés quel que soit le degré de cuisson du lait [21].

\subsection{L'ITO transforme la prise en charge}

L'ITO, en particulier au lait cuit, est un important changement dans le paradigme du traitement de l'allergie alimentaire aux protéines du lait de vache. C'est une avancée qui a 
Gâteau au yaourt

Ingrédients pour 6 personnes (environ $24 \mathrm{ml}$ de lait par part)

1 yaourt nature classique garder le pot de yaourt vide pour mesurer les autres ingrédients : farine ( 1 pot $)$, sucre (1 pot), huile (1/3 de pot)

1 sachet de levure chimique

3 œufs

Le zeste d' $1 / 2$ orange ou d' 1 citron ou fleur d'oranger ou chocolat sans protéine de lait de vache ou des fruits coupés en morceaux

Casser les œufs, ajouter le sucre et fouetter jusqu'à ce que le mélange blanchisse

Verser l'huile en filet en continuant de mélanger

Ajouter le zeste ou autre parfum, le yaourt, la farine et la levure en dernier

Bien mélanger pour obtenir une pâte homogène

Huiler le moule verser la pâte dedans

Faire cuire à four chaud $180^{\circ} 40 \mathrm{mn}$

Gâteau aux pommes

Ingrédients pour 6 personnes (environ $16 \mathrm{~mL}$ de lait par part)

Farine : 5 cuillères à soupe

Sucre : 4 cuillères à soupe +1 sachet de sucre vanillé

Lait : $100 \mathrm{~mL}$

$1 / 2$ sachet de levure chimique ou 2 cuillères à café

3 œufs

3 cuillères à soupe d'huile

3 pommes coupées en morceaux

Mettre la farine dans un saladier, ajouter la levure chimique et le lait. Bien mélanger

Casser les œufs, ajouter le sucre et fouetter jusqu'à ce que le mélange blanchisse

Ajouter le mélange œufs et sucre à la farine

Battre le tout ajouter 3 cuillères à soupe d'huile + les pommes

Huiler le moule verser la pâte dedans

Faire cuire à four chaud $180^{\circ} 45 \mathrm{mn}$

Muffins aux pépites de chocolat

Ingrédients pour 6 muffins (environ $19 \mathrm{ml}$ de lait par muffins)

Farine : $120 \mathrm{~g}$

Sucre semoule : $60 \mathrm{~g}$

Lait : $100 \mathrm{~mL}$

$1 / 2$ sachet de levure chimique ou 2 cuillères à café

1 œuf +1 blanc d'œuf

$70 \mathrm{~g}$ de beurre salé

$70 \mathrm{~g}$ de pépites de chocolat sans protéine de lait de vache

Préchauffez le four à $180^{\circ}$ (th : 5/6)

Dans un saladier, mettre la farine et la levure chimique

Ajoutez le beurre et le sucre, mélangez le tout

Mettre l'œuf, le blanc et le lait préalablement tiédi et mélanger afin d'obtenir une pâte lisse

Versez les pépites de chocolat dans la préparation et mélangez de nouveau

Remplissez à mi-hauteur les moules à muffins et mettre au four pendant $30 \mathrm{mn}$

Démoulez tiède

Vous pouvez remplacer les pépites de chocolat par des myrtilles, des framboises ou 2 cuillères à soupe de caramel liquide

Gâteau au yaourt sans ouf

Ingrédients pour 6 personnes $(50 \mathrm{~mL}$ de lait par part)

2 yaourts nature de $125 \mathrm{~g}$

1 pot de yaourt de sucre en poudre

3 pots de yaourt de farine

1 sachet de levure chimique

1 sachet de sucre vanillé

1 pincée de sel

$1 / 2$ pot d'huile

Videz vos pots de yaourt dans un saladier. Lavez et séchez en un pour l'utiliser comme doseur

Préchauffez votre four à $180^{\circ}$ pendant 10 minutes

Beurrez et farinez votre moule

Prenez votre pot de yaourt, mesurez les ingrédients et ajoutez-les dans le saladier en mélangeant progressivement jusqu'à l'obtention d'une pâte lisse

Versez la pâte dans le moule et enfournez à 180 d pendant 35 à 40 minutes.

Vérifiez la cuisson à l'aide d'un couteau. Sa lame doit ressortir sèche lorsque le gâteau est prêt

transformé la prise en charge des enfants présentant cette allergie dans ses formes persistantes. Elle améliore considérablement la qualité de vie des patients et de leur famille, sous réserve de consommer tous les jours l'aliment allergisant. Tous les patients ne peuvent toutefois en bénéficier et cette ITO, y compris au lait cuit, présente des risques. Elle ne peut donc pas être généralisée, les patients pouvant en bénéficier doivent être sélectionnés dans une évaluation permanente du bénéfice et des risques [15]. 


\section{Méthode}

Donner quotidiennement l'aliment prévu en suivant la progression décrite dans le tableau

La réintroduction doit être faite par les parents et sous leur surveillance pendant les deux heures suivant la prise de l'aliment

L'aliment ne doit donc pas être donné le soir au coucher ni avant de partir à l'école

La trousse de médicaments d'urgence doit être complète et disponible à proximité

Différents facteurs peuvent, lors de la prise de l'aliment, faciliter la survenue d'effets secondaires

Effort physique (pas d'effort important dans les 2 heures suivant la prise de l'allergène)

Forte exposition pollinique

Stress important

Infection aiguë, fièvre

Anti-inflammatoires non stéroïdiens (par exemple Advil ${ }^{\circledR}$ )

Certains médicaments antireflux (inhibiteurs de la pompe à protons)

Rupture de routine (voyage, déplacement, carence de sommeil)

Asthme mal contrôlé

Il est utile de connaître ces différentes situations

Pour analyser un incident éventuel

Pour reporter la prise de l'aliment jusqu'à la disparition des facteurs augmentant la réaction allergique

Si vous n'avez pas pu donner la dose de lait pendant 3 jours, vous pouvez donner la moitié de la dose habituelle le $4^{\mathrm{e}}$ et $5^{\mathrm{e}}$ jour puis la dose

entière à partir du $5^{\mathrm{e}}$ jour

Si vous n'avez pas pu donner la dose de lait de vache pendant plus de 4 jours contacter le médecin référent

En cas de réaction

En cas de réaction non sévère (réaction cutanée localisée ou symptômes digestifs modérés ET absence de signe respiratoire ou de retentissement

général) lors de l'augmentation des doses de lait ou laitage

Traiter la réaction en suivant le plan d'action écrit qui vous a été remis, noter les circonstances de survenue et les signes

Ne pas oublier de signaler l'incident lors de la prochaine consultation

Le lendemain revenir au palier précédent (celui qui a été toléré), à répéter de nouveau tous les jours pendant 15 jours

Puis essayer de reprendre la progression initialement prévue

En cas de réaction sévère (réaction respiratoire, réaction touchant plusieurs organes, retentissement général)

Traiter la réaction en suivant le plan d'action écrit qui vous a été remis

Noter les circonstances de survenue et les signes

Arrêter complètement l'introduction du lait de vache, contacter le médecin référent pour discuter la suite du protocole

\section{Prise en charge en pratique}

La prise en charge d'un nourrisson ou enfant présentant une APLV persistante dépend de plusieurs facteurs :

- type d'allergie alimentaire, IgE ou non IgE médiée ;

- réaction lors d'éventuelles prises accidentelles ;

- âge de l'enfant ;

- évolution des tests cutanés et des IgE spécifiques ;

- souhait de la famille ;

- capacité des parents à mener le traitement proposé ;

- effets secondaires quand l'ITO est initiée.

Plusieurs solutions sont possibles :

- poursuite de l'éviction, et ré-évaluer à distance ;

- TPO au lait cru en milieu hospitalier ;

- TPO au lait cuit en milieu hospitalier ;

- introduction d'une quantité précisée sous une forme définie à ne pas dépasser ;

- introduction progressive au domicile ;

- ITO ;

- arrêt de l'ITO en raison de la mauvaise tolérance ou observance.

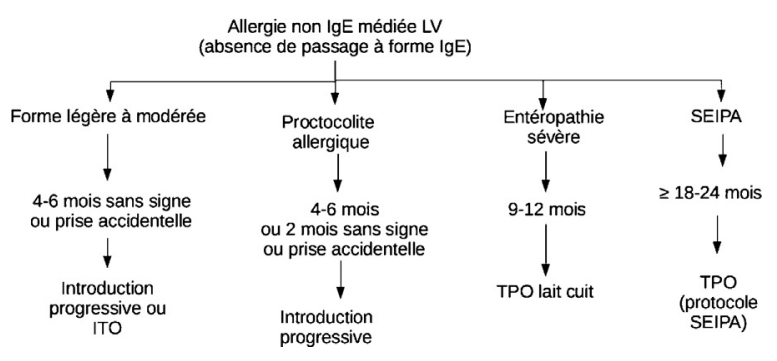

Fig. 1. Réintroduction allergie non IgE médiée.

\subsection{Allergie non IgE médiée}

\subsubsection{En cas d'allergie non IgE médiée aux PLV à forme légère à modérée}

Le régime d'éviction est maintenu pendant 4-6 mois, puis un test de réintroduction peut être proposé (Fig. 1). Au préalable, il est nécessaire de vérifier qu'il n'existe pas un passage à une forme IgE médiée. Il faut donc pratiquer un prick-test lait de vache et/ou un dosage d'IgE spécifiques lait de vache. En cas d'apparition d'une sensibilisation, ou d'apparition de manifestations IgE médiées lors d'une prise accidentelle, il faut suivre la démarche de prise en charge d'une allergie IgE médiée, avec pratique d'un TPO en milieu hospitalier. 
En l'absence de conversion en forme IgE médiée la réintroduction progressive au domicile est possible après 4-6 mois sans signe.

\subsubsection{En cas de proctocolite allergique}

Les mêmes précautions que les formes non IgE médiées légères à modérées doivent être prises avant la réintroduction.

La réintroduction progressive au domicile est possible rapidement, dès l'âge de 4 mois, ou 2 mois après les premiers symptômes. En cas de récidive des symptômes digestifs, le régime d'éviction est repris 2-3 mois avant un nouvel essai.

\subsubsection{En cas d'entéropathies allergiques aux PLV sévères}

On attendra l'âge de 9-12 mois ou au moins 6 mois de régime d'exclusion (en cas de diagnostic tardif) avant de tenter la réintroduction. Cette durée peut être réduite en cas de prise accidentelle sans réaction. Les modalités de cette réintroduction seront précisées au cas par cas, un avis en centre spécialisé s'avère nécessaire. Il peut être discuté un TPO, plutôt au lait cuit.

\subsubsection{En cas de SEIPA aux PLV}

Le régime d'éviction des PLV est le plus souvent maintenu jusqu'à l'âge de 18-24 mois. Cette durée peut être raccourcie en cas de prise accidentelle sans réaction. Les modalités du TPO au lait cru sont propres au SEIPA [7]. En cas de positivité, le régime est poursuivi. Un nouveau TPO est tenté 6-12 mois plus tard, en fonction de la sévérité des manifestations lors du premier TPO.

En cas de SEIPA avec sensibilisation IgE médiée, le protocole de TPO au lait, le plus souvent cru, est celui d'une allergie IgE médiée, mais la surveillance après la dernière prise est prolongée, et au moins de 4 à 6 heures.

\subsection{Allergie IgE médiée}

En cas d'allergie IgE médiée aux PLV prouvée par un TPO, ou pour laquelle il une existe une conviction diagnostique suffisante, le régime d'éviction des PLV est institué pour au moins 6 mois, et jusque l'âge de 9-12 mois (Fig. 2).

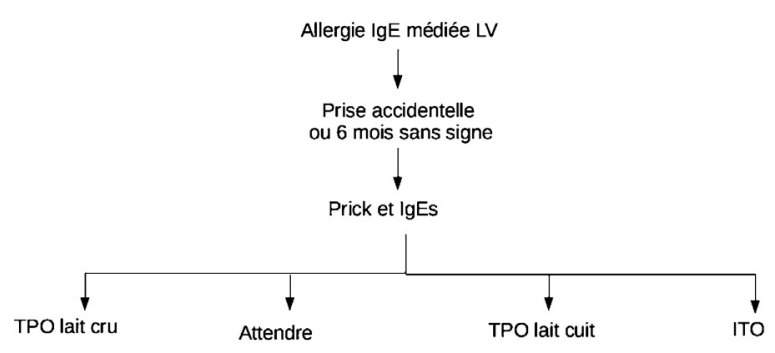

LV : lait de vache ; IgEs : IgE spécifiques ; TPO : test de provocation par voie orale.

Fig. 2. Réintroduction allergie IgE médiée.

\subsection{1. $I^{\text {re }}$ situation : prise accidentelle sans réaction}

Une prise accidentelle de LV, sans réaction, donne une information sur une quantité tolérée en vraie vie. Il peut s'agir d'un indice pour initier l'introduction du lait, et le cas échéant débuter une ITO dont les modalités seront discutées au cas par cas, éventuellement au domicile, en s'aidant d'un des tableaux d'équivalence en lait (Tableaux 8 et 9). La reprise d'un produit contenant du lait se fait à un niveau inférieur à celui de la prise accidentelle. L'âge de l'enfant, son histoire, l'évolution de la taille des prick-tests et des IgE au lait de vache peuvent guider l'indication et les modalités de la réintroduction progressive ou de l'ITO.

La possibilité d'introduire une quantité définie de PLV sans nécessairement de progression améliorera déjà la qualité de vie et favoriserait l'accélération de la guérison.

\subsection{2. $2^{e}$ situation: six mois sans symptôme allergique}

Après 6 mois sans symptôme allergique et sans prise accidentelle, l'APLV IgE médiée est réévaluée par la pratique des tests cutanés et le dosage des IgE spécifiques.

4.2.2.1. Si l'évolution du profil de sensibilisation est très favorable. Si l'évolution du profil de sensibilisation est très favorable, surtout si les manifestions d'APLV initiales n'étaient pas sévères, il est possible de faire d'emblée un TPO au lait cru.

En cas de TPO négatif, la réintroduction est progressive mais rapide au domicile.

En cas de réaction au TPO, en fonction de la dose réactogène et des manifestations survenues, on pourra proposer, une introduction du lait au domicile en débutant toujours à un niveau inférieur à la dose qui a déclenché la réaction lors du TPO, ou une progression par paliers réalisés en milieu hospitalier. En cas de réaction pour des doses non infimes de lait cru, une ITO au lait cuit est possible sans TPO lait cuit préalable.

Dans les formes initialement révélées par une anaphylaxie ou en cas de prise accidentelle récente de lait cru avec réaction, un TPO lait cuit est souvent proposé en $1^{\text {re }}$ intention.

\subsubsection{Si l'évolution des tests allergologiques est défavorable.} Si l'évolution des tests allergologiques est défavorable, c'est-àdire lorsque la sensibilisation augmente, tout spécialement les IgE pour la caséine, cela impose de sursoir à tout TPO et de reproduire ces examens 6 mois plus tard, surtout si le nourrisson a $12-18$ mois.

4.2.2.3. Chez les enfants plus âgés. Chez les enfants plus âgés, malgré une évolution défavorable du profil de sensibilisation, il est possible de tenter un TPO au lait cuit en milieu hospitalier. Un TPO au lait cuit est aussi possible si le profil de sensibilisation est stable ou en faible diminution ou dans les situations à haut risque. Il faut rappeler toutefois que le risque de réaction sévère existe dans ces situations pour les deux formes de lait, cru et cuit.

Si la dose réactogène et la nature de la réaction observées en TPO le permettent, une ITO est à discuter au cas par cas, suivant un des tableaux de lait (Tableaux 8 et 9). Dans les formes 
plus sévères, l'augmentation des doses pourra se faire en milieu hospitalier.

4.2.2.4. Une ITO au domicile, sans TPO préalable, est très exceptionnellement proposée dans les allergies IgE médiées en l'absence de prise accidentelle. Une ITO au domicile, sans TPO préalable, est très exceptionnellement proposée dans les allergies IgE médiées en l'absence de prise accidentelle. Elle peut être discutée en cas de manifestations initiales mineures, si le profil de sensibilisation s'améliore de manière spectaculaire, voire se négative. On débute habituellement avec des faibles doses de lait cuit.

\subsection{Comment faire une réintroduction progressive rapide au domicile?}

Pour les allergies non IgE-médiées non sévères, la réintroduction peut se faire directement au domicile, de manière progressive sur quelques jours à semaines. Il ne faut pas réaliser de réintroduction brutale, car après quelques mois de régime d'éviction, le lait de vache peut être mal toléré, avec des troubles digestifs, et aboutir à un arrêt précoce de la réintroduction, non justifié. De plus, l'enfant habitué au goût de son hydrolysat pourrait refuser le lait infantile si la réintroduction est trop rapide.

Plusieurs schémas d'introduction progressive du lait sont possibles, en fonction de l'âge de l'enfant. Pour tous les enfants, il est prudent de débuter par le lait cuit sous forme de Véritable Petit Beurre de Lu ${ }^{\circledR}$ puis le beurre.

Chez le petit, il est ensuite possible de substituer une mesure du lait de régime par une mesure d'un lait adapté à l'âge de l'enfant. Si la tolérance est bonne, une mesure par biberon est introduite, puis par palier de 1 à 3 jours, une mesure supplémentaire de lait est substituée à chaque fois. La réintroduction complète se fait ainsi en 1 semaine à 1 mois.

Chez le plus grand, il est possible de suivre l'échelle de lait en étant rapide dans les paliers. Une fois tous les produits à base de lait « cuit » puis fermentés tolérés, le lait cru est introduit en mélangeant le lait de régime et le lait adapté à l'âge de l'enfant (3/4 lait de régime $+1 / 4$ lait adapté à l'âge, puis $1 / 2-1 / 2$, puis 3/4-1/4, puis lait adapté à l'âge de l'enfant uniquement).

En cas de réaction modérée avec des troubles digestifs ou un eczéma, il ne faut pas arrêter, mais diminuer la dose puis augmenter un peu plus progressivement.

\section{Déclaration de liens d'intérêts}

Pour tous les auteurs : intervention ponctuelle, Laboratoires Mead Jonhson pour le support à l'organisation dans la rédaction de ce guide.

E. B. Interventions ponctuelles conférences Laboratoires DBV, Nutricia, Mead Jonhson.

A. D. Interventions ponctuelles Laboratoires DBV, Mead Jonhson.

J. L. Interventions ponctuelles Laboratoires Lactalis, Nutricia, Mead Jonhson.
P.T. Interventions ponctuelles Laboratoires Blédina, Mead Johnson, Nestlé, Novalac, Nutricia, Sodilac.

Pour tous les autres auteurs : Intervention ponctuelle, Laboratoires Mead Jonhson pour le support à l'organisation dans la rédaction de ce guide.

\section{Remerciements}

Aux laboratoires Mead Jonhson qui ont facilité la rédaction de ce guide.

\section{Références}

[1] Venter C, Brown T, Meyer R, et al. Better recognition, diagnosis and management of non-IgE-mediated cow's milkallergy in infancy: iMAPan international interpretation of the MAP (Milk Allergy in Primary Care) guideline. Clin Transl Allergy 2017;7:26, http://dx.doi.org/10. 1186/s13601-017-0162-y [eCollection].

[2] Luyt D, Ball H, Makwana N, et al. Standards of Care Committee (SOCC) of the British Society for Allergy and Clinical Immunology (BSACI). BSACI guideline for the diagnosis and management of cow's milk allergy. Clin Exp Allergy 2014;44:642-72.

[3] Schoemaker AA, Sprikkelman AB, Grimshaw KE, et al. Incidence and natural history of challenge-proven cow's milk allergy in European children-EuroPrevall birth cohort. Allergy 2015;70:963-72.

[4] NIAID-Sponsored Expert Panel., Boyce JA, Assa'ad A, Burks AW, et al Guidelines for the diagnosis and management of food allergy in the United States: Report of the NIAID-sponsored expert, panel. J Allergy Clin Immunol 2010;126(Suppl):S1-58.

[5] Sicherer SH, Sampson HA. Food allergy: A review and update on epidemiology, pathogenesis, diagnosis, prevention, and management. J Allergy Clin Immunol 2018;141:41-58.

[6] Garcette K. Réintroduction des protéines du lait de vache 2017. http://pappediatrie.fr/allergo-pneumo/reintroduction-des-proteines-de-lait-de-vache (consulté le 4 novembre 2018).

[7] Nowak-Węgrzyn A, Chehade M, Groetch ME, et al. International consensus guidelines for the diagnosis and management of food protein-induced enterocolitis syndrome: Executive summary-Workgroup Report of the Adverse Reactions to Foods Committee, American Academy of Allergy, Asthma \& Immunology. J Allergy Clin Immunol 2017;139:1111-26 [Ces recommandations sont en accès libre sur internet] http://www.jacionline. org/article/S0091-6749(17)30153-7/pdf elles citent 104 références.

[8] Bidat É, Benoist G. Le syndrome d'entérocolite induite par les protéines alimentaires. Presse Med 2017;46:263-70.

[9] Nowak-Wegrzyn A, Bloom KA, Sicherer SH, et al. Tolerance to extensively heated milk in children with cow's milk allergy. J Allergy Clin Immunol 2008;122:342-7.

[10] Sackesen C, Suárez-Fariñas M, Sillva R, et al. A new Luminex-based peptide assay to identify reactivity to baked, fermented, and whole milk. Allergy 2018, http://dx.doi.org/10.1111/all.13581.

[11] Bidat É. Allergie alimentaire : l'immunothérapie, un changement dans le paradigme du traitement. mt pédiatrie 2017;20:129-35.

[12] Brożek JL, Terracciano L, Hsu J, et al. Oral immunotherapy for IgEmediated cow's milk allergy: a systematic review and meta-analysis. Clin Exp Allergy 2012;42:363-74.

[13] Cuomo B, Indirli GC, Bianchi A, et al. Specific IgE, skin prick tests to diagnose allergy to fresh, baked cow's milk according to age: a systematic review. Ital J Pediatr 2017;43:93

[14] Leonard SA, Caubet JC, Kim JS, et al. Baked milk- and egg-containing diet in the management of milk and egg allergy. J Allergy Clin Immunol Pract 2015;3:13-23.

[15] Bidat É, Tressol C, Benoist G, Feuillet-Dassonval C. Immunothérapie orale au lait de vache cuit, aspects pratiques. Rev Fr Allergol 2016;56:372-7.

[16] Niggemann B, Beyer K. Factors augmenting allergic reactions. Allergy 2014;69:1582-7. 
[17] Venter C, Brown T, Shah N, et al. Diagnosis and management of non-IgEmediated cow's milk allergy in infancy - a UK primary care practical guide. Clin Transl Allergy 2013;3:23, http://dx.doi.org/10.1186/2045-7022-3-23.

[18] Pajno GB, Fernandez-Rivas M, Arasi S, et al. EAACI Allergen Immunotherapy Guidelines Group. EAACI Guidelines on allergen immunotherapy: IgE-mediated food allergy. Allergy 2018;73:799-815.

[19] Dupont C, Soulaines P. Actualités thérapeutiques dans la prise en charge nutritionnelle de l'allergie aux protéines de lait de vache. Arch Pediatr 2017;24:1350-7.
[20] Lucendo AJ, Arias A, Tenias JM. Relation between eosinophilic esophagitis and oral immunotherapy for food allergy: a systematic review with meta-analysis. Ann Allergy Asthma Immunol 2014;113: 624-9.

[21] Amat F, Kouche C, Gaspard W, et al. Is a slow-progression baked milk protocol of oral immunotherapy always a safe option for children with cow's milk allergy? A randomized controlled trial. Clin Exp Allergy 2017:47:1491-6. 